\begin{tabular}{|c|l|}
\hline Title & $\begin{array}{l}\text { Fabrication of InP/nA s/nP core multishell heterostructure nanowires by selective area metal organic vapor phase } \\
\text { epitaxy }\end{array}$ \\
\hline Author(s) & Mohan, Premila; Motohisa, Junichi; Fukui, Takashi \\
\hline Citation & $\begin{array}{l}\text { A pplied Physics Letters, 88, 133105 } \\
\text { https://doi.org/_0.1063/2189203 }\end{array}$ \\
\hline Issue Date & 2006-03_27 \\
\hline Doc URL & http://hdl.handle.net/2115/8416 \\
\hline Rights & Copyright $\odot 2006$ A merican Institute of Physics. \\
\hline Type & article \\
\hline File Information & A pplPhysLett_88_133105.pdf \\
\hline
\end{tabular}

Instructions for use 


\title{
Fabrication of InP/InAs/InP core-multishell heterostructure nanowires by selective area metalorganic vapor phase epitaxy
}

\author{
Premila Mohan, ${ }^{\text {a) }}$ Junichi Motohisa, and Takashi Fukui \\ Research Center for Integrated Quantum Electronics, Graduate School of Information Science \\ and Technology, Hokkaido University, North 13 West 8, Sapporo 060-8628, Japan
}

(Received 2 November 2005; accepted 16 January 2006; published online 27 March 2006)

\begin{abstract}
We report the growth of $\mathrm{InP} / \mathrm{InAs} / \mathrm{InP}$ core-multishell nanowire arrays by selective area metalorganic vapor phase epitaxy. The core-multishell nanowires were designed to accommodate a strained InAs quantum well layer in a higher band gap InP nanowire. The precise control over nanowire growth direction and heterojunction formation enabled the successful fabrication of the nanostructure in which all three layers were epitaxially grown without the assistance of any catalyst. The grown nanowires were highly uniform, vertically oriented, and periodically aligned with controllable dimensions. $4 \mathrm{~K}$ photoluminescence measurements confirmed the formation of strained InAs quantum well on InP (110) sidewalls and the well widths corresponding to the photoluminescence peaks were in good agreement with calculated values. (C) 2006 American Institute of Physics. [DOI: 10.1063/1.2189203]
\end{abstract}

Semiconductor nanowires are potential building blocks for future nanoscale electronic and photonic devices due to their superior properties and quantum confinement effects. Active nanoscale devices such as field effect transistors, ${ }^{1}$ logic circuits, ${ }^{2}$ sensors, ${ }^{3}$ lasers, ${ }^{4}$ and light-emitting diodes ${ }^{5}$ based on individual homogeneous nanowires have been demonstrated. The potential ability of these semiconductor nanowires can be greatly enhanced by introducing heterostuctures within nanowires and recently significant advances have been made on the synthesis of axial heterostructure nanowires and radial heterostructure or core-shell nanowires. While core-shell nanowires enable passivation of interface states thereby improving the overall performance of the resultant semiconductor devices, core-multishell nanowires formed by sequential modulation of composition along the radial direction have the distinct ability to incorporate multifunction into an individual nanowire. In spite of the potential impact, core-multishell nanowires are virtually unexplored with the exception of very few reports, ${ }^{6-8}$ since the fabrication is extremely challenging due to the complexity involved.

In this letter, we report the fabrication of an innovative core-multishell nanowire that was designed to embed a single strained radial quantum well (QW) in a higher band gap nanowire. The InP/InAs material system with a lattice mismatch of $3.2 \%$ was chosen for the present work. The InP/InAs strained QW has tremendous potential for long wavelength optoelectronic applications because of their flexibility for tailoring the electronic band structure by the combined effects of quantum confinement and strain. Schematic illustration of the proposed core-multishell nanowire is shown in Fig. 1(a). The layer structure consists of inner InP core and InAs and InP inner and outer shells, respectively, in which the InP core and the outer InP shell serve as the barrier layers while the InAs shell is the strained QW layer. Since the active layer, which is the InAs strained QW, is in the form of a hollow cylindrical tube, this nanostructure can be

\footnotetext{
${ }^{\text {a) }}$ Author to whom correspondence should be addressed; electronic mail:
} pmohan@ rciqe.hokudai.ac.jp reckoned as a quantum nanotube and is likely to show exceptional properties as it is a two-dimensionally (2D) confined system with three-dimensional (3D) cylindrical symmetry within a one-dimensional (1D) nanowire structure.

In this work, we employed selective area metalorganic vapor phase epitaxy (SA-MOVPE) that has been established recently as a versatile method to realize well controlled nanowires without involving any catalyst. ${ }^{9,10}$ Fabrication of the lattice mismatched core-multishell heterostructure nanowire is highly challenging due to several complex requirements. In spite of the lattice mismatch, InAs 2D layer-bylayer growth should essentially occur on the InP nanowire
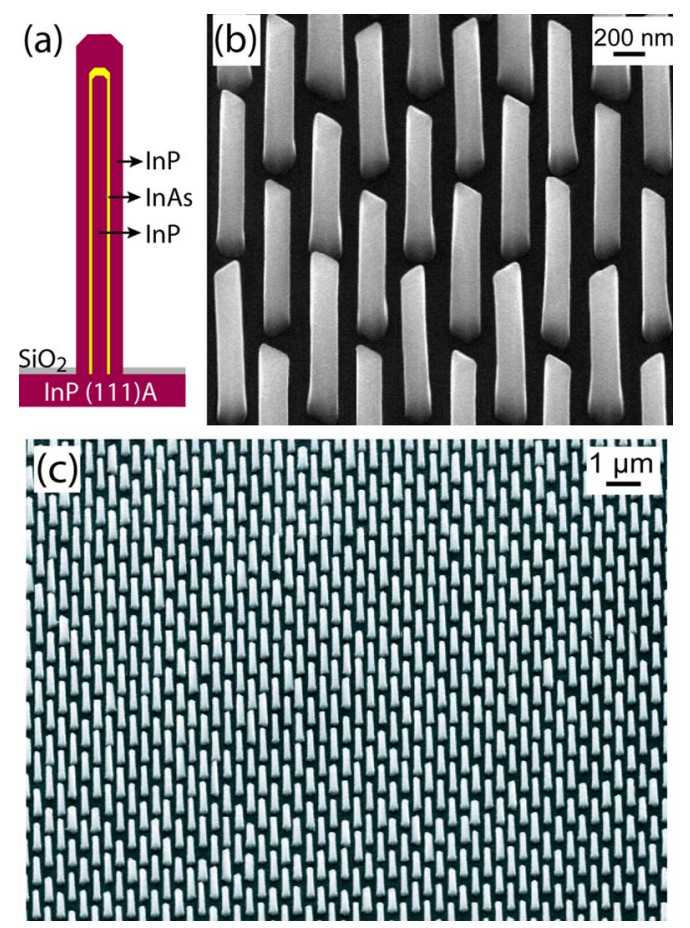

FIG. 1. (Color online) (a) Schematic cross-sectional image of InP/InAs/InP core-multishell nanowire. (b) SEM image of periodically aligned InP/InAs/InP core-multishell nanowire array. (c) Low-angle inclined SEM image showing high dense ordered arrays of core-multishell nanowires. 
core and that too preferentially along the lateral direction. Further, the fact that the inner core and the outermost shell are composed of the same material, InP, intrinsically necessitates InP to be grown axially for the inner core and laterally for the outer shell. The core-multishell nanowires reported earlier based on $\mathrm{Si} / \mathrm{Ge}$ (Ref. 6) and GaN/InGaN (Refs. 7 and 8) have been synthesized by a different approach, in which case the nanowire core was grown by the catalyst-assisted vapor-liquid-solid (VLS) method and the shells were formed on the nanowire surface by vapor phase deposition. Unlike such synthesis, our work involves the growth of both the core and the shells by pure epitaxial method without the assistance of any catalyst. The accurate control over the fabrication process, precise knowledge of the growth mechanism, and command over the growth direction are the critical factors essential to accomplish such a difficult task.

Selective area MOVPE growth of InP/InAs/InP coremultishell nanowires was carried out on InP (111)A patterned substrates with hexagonal mask openings using a lowpressure horizontal MOVPE system and the source materials were trimethylindium (TMI), tertiarybutylphosphine (TBP), and $5 \%$ arsine $\left(\mathrm{AsH}_{3}\right)$ in hydrogen. The growth of the core as well as the shells was performed simultaneously as a single-step process. The growth conditions of InP core and InAs shell have been described in detail elsewhere. ${ }^{11,12}$ Subsequent to the InAs growth, the conditions were changed yet again for the growth of InP outer shell. While the growth of the inner InP core occurred along the top $\langle 111\rangle$ direction, the outer InP layer should preferentially grow along the $\langle 110\rangle$ direction. The control of axial/radial growth direction of InP is essential to achieve this complicated task. Phosphorus (P) coverage governed by the growth temperature and TBP partial pressure was found to strongly influence the InP growth direction. ${ }^{11}$ While relatively lower $\mathrm{P}$ coverage induced axial growth, higher $\mathrm{P}$ coverage influenced lateral growth. Thus, as $P$ coverage was altered, a competing growth of the top (111)A surface and the $\{110\}$ sidewall plane occurred and by precisely choosing the conditions it was possible to exactly define the growth direction. Growth temperature of $600{ }^{\circ} \mathrm{C}$ and high TBP partial pressure of $5.5 \times 10^{-3}$ atm were the optimum conditions that favored the lateral growth of $\mathrm{InP}$ over InAs sidewall facets. The single-step growth process with the above mentioned conditions ultimately resulted in the successful fabrication of InP/InAs/InP core-multishell heterostructure nanowire arrays.

Scanning electron microscopy (SEM) studies clearly indicated that the grown structures were well defined and extremely uniform [Figs. 1(b) and 1(c)]. The length of a typical core-multishell nanowire was about $2.5 \mu \mathrm{m}$ and the diameter was about $140 \mathrm{~nm}$. The grown nanowires were subjected to anisotropic dry etching followed by stain etching to analyze their cross-sectional features. The diameter of the inner InP core and the thickness of the outer InP shell were 70 and $30 \mathrm{~nm}$, respectively. The InAs QW layer exhibited a welldefined hexagonal cross section, and the well width was found to be $5 \mathrm{~nm}$ (Fig. 2). The remarkable feature of this nanostructure is that the dimensions as well as the layer structure thickness can be accurately defined by growth conditions. Thus, precise control over the fabrication process gives us the freedom to engineer the physical properties determined by the shape, size, and QW width of the nanostructure. Further, the selective area growth guided the fabrication of these nanowires in a highly periodic manner. The success-

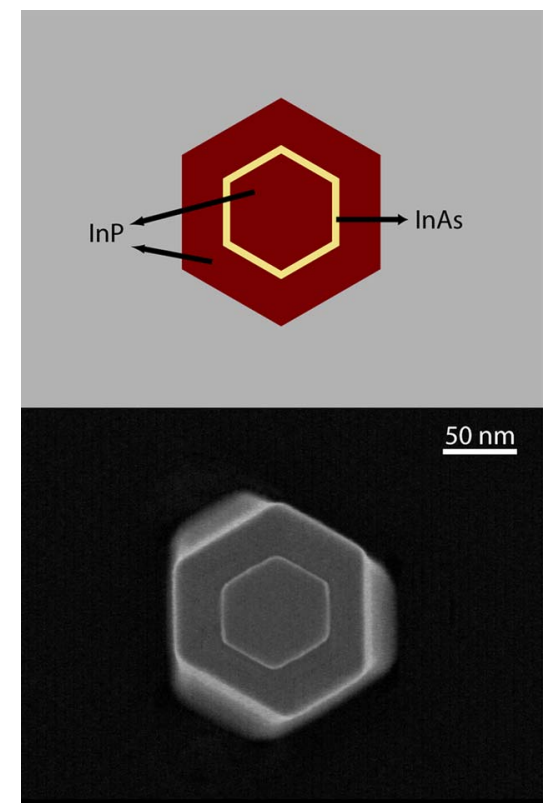

FIG. 2. (Color online) Schematic illustration and high resolution SEM cross-sectional image of a typical core-multishell nanowire observed after anisotropic dry etching and stain etching.

ful fabrication of this core-multishell nanowire assumes profound significance as all three layers are single crystalline and epitaxially grown without the presence of any catalyst. For further characterization, core-multishell nanowires with different InAs QW widths were grown by varying the InAs growth time.

$4 \mathrm{~K}$ microphotoluminescence $(\mu \mathrm{PL})$ measurements were carried out on the core-multishell nanowires with InAs well width of $1.5 \mathrm{~nm}$ using a $532 \mathrm{~nm}$ continuous-wave output of a diode pumped $\mathrm{Nd}: \mathrm{YVO}_{4}$ laser for excitation. The excitation beam was focused onto $\sim 2 \mu \mathrm{m}$ diam spot with a $\times 20$ microscope objective on the sample placed in a coldfinger cryostat. The PL collected through the same microscope objective was dispersed into a spectrometer with a liquid-nitrogen-cooled InGaAs photomultiplier tube. PL spectra of core-multishell nanowires with different pattern periods are shown in Fig. 3. Spectrum (a) shows PL from a nanowire array with $3 \mu \mathrm{m}$ period, whereas (b) is from an array with $0.4 \mu \mathrm{m}$ period. Since the $3 \mu \mathrm{m}$ period is greater

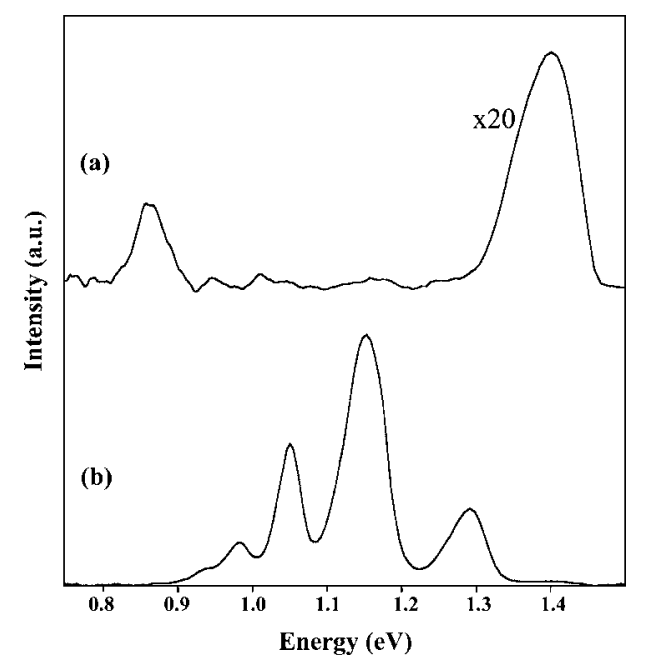

FIG. 3. $4 \mathrm{~K}$ PL spectra of nanowire arrays with (a) 3 and (b) $0.4 \mu \mathrm{m}$ periods, respectively.

o AlP license or copyright, see http://apl.aip.org/apl/copyright.jsp 


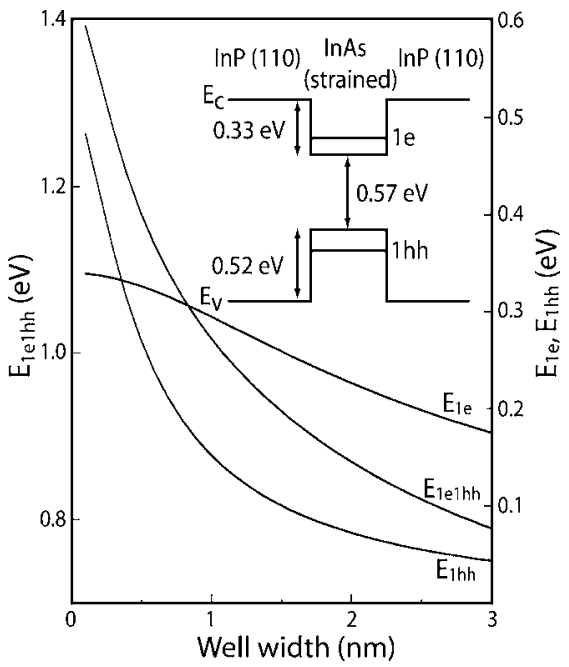

FIG. 4. Plot of ground-state transition energy as a function of well width for InP/InAs strained QW on InP (110).

than the spot size, the spectrum (a) can be concluded to be from a single core-multishell nanowire. ${ }^{13}$ The spectrum (a) consisted of two distinct emission peaks. The PL peak observed at energy of $0.861 \mathrm{eV}$ is due to InAs QW formed on the (110) sidewalls of InP nanowires, while the peak at $1.401 \mathrm{eV}$ corresponds to the InP barrier, as discussed in detail later. Further, the InAs layer on top of the InP core is rather thick and does not have any influence on the observed PL spectra. The spectrum (b), taken from multiple coremultishell nanowires $(\sim 30$ nanowires considering the spot size), on the other hand, exhibits multiple peaks at around $0.86-1.45 \mathrm{eV}$, which are also due to the emission from InAs radial QWs.

To confirm these peak assignments, the ground-state transition energy in strained InAs QWs on InP (110) was calculated using a simple square well potential. The parameters for calculation were determined as follows. Initially, offsets of conduction and heavy hole band were calculated based on the model solid theory ${ }^{14}$ taking into account strain in InAs on InP (110). While the unstrained isotropic effective mass was used for electrons, the heavy hole effective mass $\left(m_{\mathrm{hh}}\right)$ was determined based on the following expression:

$$
m_{\mathrm{hh}}=\frac{m_{0}}{\gamma_{1}-\sqrt{\gamma_{2}^{2}+3 \gamma_{3}^{2}}},
$$

where $m_{0}$ is the bare electron mass and $\gamma_{1}, \gamma_{2}$, and $\gamma_{3}$ are the Luttinger parameters. The heavy hole effective mass was obtained using $4 \times 4$ Luttinger-Kohn Hamiltonian with quantization axis along the [110] direction. ${ }^{15}$ Values of deformation potential and Luttinger parameters were taken from Refs. 16 and 17. Present calculation considerably simplifies the treatment of nonparabolicity of the conduction band and complex valence band structure of strained (110)-oriented QWs. A more accurate argument on the electronic structure in strained core-multishell nanowire will be reported elsewhere, but the accuracy of the present theoretical treatment is expected to be within the ambiguity of the parameters, the difference in the crystallographic structure ${ }^{12}$ of InP and InAs, and the effective-mass approximation, and so on. The results of the calculation are plotted as a function of InAs well width (Fig. 4). According to these results, the PL peak at $0.861 \mathrm{eV}$ observed for a single core-multishell nanowire corresponds to a well width of $2.08 \mathrm{~nm}$, which reasonably agrees with the measured thickness of the InAs layer. Further, this peak is relatively broader, full width at half maximum (FWHM) is $48 \mathrm{meV}$, which can be mainly accounted to the fluctuation of the well width, although it may be in part due to the inhomogeneous strain in the InAs layer. It may also be noted that the PL peak of InP is slightly redshifted as compared to the bare InP nanowires. ${ }^{11}$ Our preliminary calculations suggest that this shift is not due to the tensile strain in InP nanowires and it requires further investigation. In spectrum (b) the peaks at $0.984,1.05,1.154$, and $1.292 \mathrm{eV}$ correspond to InAs QW widths of $1.16,0.856,0.532$, and $0.262 \mathrm{~nm}$, respectively. Minimum width for InAs in InP is realized by substituting one layer of $\mathrm{P}$ with As and corresponds to half monolayer thickness for (110). Thus, in the elastic continuum model, thinnest InAs QW is expected to be $0.219 \mathrm{~nm}$, and the well width should be its integer multiples. Considering the accuracy of the present model, these results reiterate the formation of InAs QWs on the sidewalls of InP nanowires and the multiple peaks indicate that there might be monolayer QW thickness variations in individual nanowires in an array. The peak broadening, in this case also, can be partly attributed to the inhomogeneous strain in the InAs layer in the nanowire array. Further, the InAs layer thickness is less in nanowires with a $0.4 \mu \mathrm{m}$ period than that of nanowires with a $3 \mu \mathrm{m}$ period. This difference in the InAs layer thickness may be attributed to the gas-phase diffusion of In, in which case the lateral growth rate of InAs on the sidewalls is much higher for smaller density of nanowires, i.e., for nanowire array with larger period. However, this kind of diffusion model cannot fully account for the vertical growth rate of nanowires, thus requiring more detailed investigation.

One of the authors (P.M.) acknowledges the Japan Society for Promotion of Science for a postdoctoral fellowship.

${ }^{1}$ Y. Cui and C. M. Lieber, Science 291, 851 (2001).

${ }^{2}$ Y. Huang, X. Duan, Y. Cui, L. J. Lauhon, K. H. Kim, and C. M. Lieber, Science 294, 1313 (2001).

${ }^{3}$ Y. Cui, Q. Wei, H. Park, and C. M. Lieber, Science 293, 1289 (2001).

${ }^{4}$ M. H. Huang, S. Mao, H. Feick, H. Yan, Y. Wu, H. Kind, E. Weber, R. Russo, and P. Yang, Science 292, 1897 (2001).

${ }^{5}$ X. Duan, Y. Huang, Y. Cui, and C. M. Lieber, Nature (London) 409, 66 (2001).

${ }^{6}$ L. J. Lauhon, M. S. Gudiksen, D. Wang, and C. M. Lieber, Nature (London) 420, 57 (2002).

${ }^{7}$ F. Qian, Y. Li, S. Gradecak, D. Wang, C. J. Barrelet, and C. M. Lieber, Nano Lett. 4, 1975 (2004).

${ }^{8}$ F. Qian, S. Gradecal, Y. Li, C. Y. Wen, and C. M. Lieber, Nano Lett. 5, 2287 (2005).

${ }^{9}$ J. Motohisa, J. Noborisaka, J. Takeda, M. Inari, and T. Fukui, J. Cryst. Growth 272, 180 (2004).

${ }^{10}$ J. Noborisaka, J. Motohisa, and T. Fukui, Appl. Phys. Lett. 86, 2132102 (2005).

${ }^{11}$ P. Mohan, J. Motohisa, and T. Fukui, Nanotechnology 16, 2903 (2005).

${ }^{12}$ P. Mohan, J. Motohisa, and T. Fukui, Appl. Phys. Lett. 88, 013110 (2006).

${ }^{13}$ S. Hara, J. Motohisa, J. Noborisaka, J. Takeda, and T. Fukui, Inst. Phys. Conf. Ser. 184, 393 (2005).

${ }^{14}$ C. G. Van de Walle, Phys. Rev. B 39, 1871 (1989); For the calculation of strain-induced shift on (110), Eq. (15) in this reference should be replaced by Eqs. (13) and (14).

${ }^{15}$ Y. Kajikawa, M. Hata, and T. Isu, Jpn. J. Appl. Phys., Part 1 30, 1944 (1991).

${ }^{16}$ S. L. Chuang, Phys. Rev. B 43, 9649 (1991).

${ }^{17}$ M. Sugawara, N. Okazaki, T. Fujii, and S. Yamazaki, Phys. Rev. B 48, 8102 (1993). 